EFI-99-20

hep-ph/9905366

May 1999

\title{
FINAL-STATE PHASES IN CHARMED MESON TWO-BODY NONLEPTONIC DECAYS \&
}

\author{
Jonathan L. Rosner \\ Enrico Fermi Institute and Department of Physics \\ University of Chicago, Chicago, IL 60637
}

\begin{abstract}
Observed decay rates indicate large phase differences among the amplitudes for the charge states in $D \rightarrow \bar{K} \pi$ and $D \rightarrow \bar{K}^{*} \pi$ but relatively real amplitudes in the charge states for $D \rightarrow \bar{K} \rho$. This feature is traced using an $\mathrm{SU}(3)$ flavor analysis to a sign flip in the contribution of one of the amplitudes contributing to the latter processes in comparison with its contribution to the other two sets. This amplitude may be regarded as an effect of rescattering and is found to be of magnitude comparable to others contributing to charmed particle two-body nonleptonic decays.
\end{abstract}

PACS codes: 13.25.Ft, 11.30.Hv, 14.40Lb

\section{INTRODUCTION}

The phases of amplitudes in weak two-body nonleptonic decays of heavy mesons are of interest in the search for $\mathrm{CP}$ violation. Decays of $B$ mesons in many cases are expected to receive contributions from more than one weak subprocess. If the corresponding amplitudes also differ in their strong phases one can expect to see CP-violating asymmetries [1] in $B$ meson decay rates. The origin and magnitude of such strong phase differences has been the subject of much discussion [2, 3].

The nonleptonic decays of charmed mesons are expected to involve weak amplitudes with very similar phases 沺. If decay amplitudes exhibit large phase differences they are almost certainly due to strong final-state interactions. Thus these decays can serve as a laboratory for the examination of final-state effects. The lower mass of charmed particles in comparison with $B$ mesons tends to amplify these effects, which are expected to diminish in relative importance with increasing energy.

One class of charmed meson decays in which final-state interactions can be wellprobed experimentally is the set of Cabibbo-favored nonleptonic two-body decays governed by the subprocess $c \rightarrow s u \bar{d}$. By comparing decay rates, one finds that the amplitudes for the three charge states $\bar{K}^{0} \pi^{+}, K^{-} \pi^{+}, \bar{K}^{0} \pi^{0}$ in $D \rightarrow \bar{K} \pi$ decays cannot all be

\footnotetext{
${ }^{1}$ To be submitted to Phys. Rev. D.
} 
real with respect to one another [5, 6, 0, 8]. A similar conclusion can be drawn both from decay rates and from relative phases of Dalitz plot amplitudes for the three charge states in $D \rightarrow \bar{K}^{*} \pi$ [5, 9, 10, 11. However, both decay rates and Dalitz plot analyses reveal no relative phases between the amplitudes in the three charge states of $D \rightarrow \bar{K} \rho$.

In the present paper we examine the source of this apparent difference between $\bar{K} \pi, \bar{K}^{*} \pi$ decays on the one hand and $\bar{K} \rho$ decays on the other. We find that effects of strong final-state interactions are present in all three decays, but they contribute to the various charge states in $\bar{K} \rho$ decays in such a way that the amplitudes are all relatively real. This result has some implications for the universality of strong finalstate-interaction effects. In passing, we note some simple regularities of contributions to these processes which can be related to those in semileptonic decays.

We consider only Cabibbo-favored decays, in order to focus on the comparison between $\bar{K} \pi, \bar{K}^{*} \pi$, and $\bar{K} \rho$. The framework we employ is an $\mathrm{SU}(3)$ flavor analysis [12, 13, 14] which can be expressed in terms of quark graphs [15, 16] but whose interpretation in those terms should not be taken too literally. We assume the following contributions: (1) A color-favored "tree" amplitude T, (2) a "color-suppressed" tree amplitude $C$, (3) an "exchange" amplitude $E$ contributing only to $D^{0}$ decays, and (4) an "annihilation" amplitude $A$ contributing only to $D_{s}$ decays. The amplitudes $T$ and $C$ do not involve the spectator quark, while $E$ and $A$ do. They are most likely parametrizations of rescattering effects, since when taken literally as short-distance operators their calculated magnitudes are too small.

Many authors [17 have recognized that $E$ and $A$ can have non-zero phases relative to $T$ and $C$. Our result, which distinguishes the present analysis from the previous ones, is that $T$ and $C$ appear to differ from one another in phase as well. This phase difference appears to be similar (about $150^{\circ}$ ) in $\bar{K} \pi, \bar{K}^{*} \pi$, and $\bar{K} \rho$ decays. It probably arises as a result from rescattering. The $E$ amplitude in $D^{0}$ decays and the $A$ amplitude in $D_{s}$ decays are found to have large phases with respect to both $C$ and $T$. We find that if the relative contributions of $T, C$, and $E$ are such as to give large relative phases between amplitudes for the three different charge states in $D \rightarrow \bar{K} \pi$ and $D \rightarrow \bar{K}^{*} \pi$, then these phases naturally cancel in $D \rightarrow \bar{K} \rho$, leaving amplitudes which are real with respect to one another. The $D \rightarrow \bar{K} \rho$ amplitudes do contain contributions from final-state interactions, but they are masked by cancelling phases. This has important implications if one wishes to ascribe final-state interactions to the proximity of resonances [6, 18].

This paper is organized as follows. In Section II we review the flavor-SU(3) decomposition of amplitudes and introduce notation for invariant amplitudes. We tabulate the processes of interest, their decay rates, and their amplitudes in Section III. Then, in Section IV, we extract reduced amplitudes from the data, and display pictorially their magnitudes and phases. The amplitudes $T$ extracted in this way are compared with predictions from factorization and semileptonic decays in Section V. A brief discussion of resonant contributions is contained in Section VI. The role of disconnected diagrams involving $\eta$ and $\eta^{\prime}$ production, which may be important in the decays $D_{s} \rightarrow \rho^{+}+\left(\eta, \eta^{\prime}\right)$, is discussed in Section VII, while Section VIII concludes. 


\section{NOTATION}

Our meson wave functions are assumed to have the following quark content, with phases chosen so that isospin multiplets contain no relative signs [16, 19]:

- Charmed mesons: $D^{0}=-c \bar{u}, D^{+}=c \bar{d}, D_{s}^{+}=c \bar{s}$.

- Pseudoscalar mesons P: $\pi^{+}=u \bar{d}, \pi^{0}=(d \bar{d}-u \bar{u}) / \sqrt{2}, \pi^{-}=-d \bar{u}, K^{+}=u \bar{s}$, $K^{0}=d \bar{s}, \bar{K}^{0}=s \bar{d}, K^{-}=-s \bar{u}, \eta=(s \bar{s}-u \bar{u}-d \bar{d}) / \sqrt{3}, \eta^{\prime}=(u \bar{u}+d \bar{d}+2 s \bar{s}) / \sqrt{6}$. (Here we adopt a specific ansatz [19] for octet-singlet mixing in the $\eta$ and $\eta^{\prime}$ wave functions.)

- Vector mesons $V: \rho^{+}=u \bar{d}, \rho^{0}=(d \bar{d}-u \bar{u}) / \sqrt{2}, \rho^{-}=-d \bar{u}, \omega=(u \bar{u}+d \bar{d}) / \sqrt{2}$, $K^{*+}=u \bar{s}, K^{* 0}=d \bar{s}, \bar{K}^{* 0}=s \bar{d}, K^{*-}=-s \bar{u}, \phi=s \bar{s}$.

The partial width $\Gamma$ for a specific two-body decay to $P P$ is expressed in terms of an invariant amplitude $\mathcal{A}$ as

$$
\Gamma(D \rightarrow P P)=\frac{p^{*}}{8 \pi M^{2}}|\mathcal{A}|^{2}
$$

where $p^{*}$ is the center-of-mass (c.m.) 3-momentum of each final particle, and $M$ is the mass of the decaying particle. The kinematic factor of $p^{*}$ is appropriate for the $\mathrm{S}$-wave final state. The amplitude will thus have dimensions of (energy) ${ }^{-1}$.

For $P V$ decays a $\mathrm{P}$-wave kinematic factor is appropriate instead, and

$$
\Gamma(D \rightarrow P V)=\frac{\left(p^{*}\right)^{3}}{8 \pi M^{2}}\left|\mathcal{A}^{\prime}\right|^{2}
$$

Here $\mathcal{A}^{\prime}$ is dimensionless. These conventions agree with those of Chau et al. [15].

\section{DECAY RATES AND AMPLITUDES}

In Tables I and II we summarize the rates, invariant amplitudes, and their flavor$\mathrm{SU}(3)$ representations for decays of charmed mesons to two pseudoscalar mesons and to one pseudoscalar and one vector, respectively. The branching ratios are taken from the compilation of Ref. [20] except for branching ratios for $D_{s} \rightarrow\left(\pi^{+}, \rho^{+}\right)+\left(\eta, \eta^{\prime}\right)$ from Ref. [21], and are converted to decay rates using charmed particle lifetimes which are averages [3] of those in Ref. [20] and new CLEO values [22]: $\tau\left(D^{+}\right)=1051 \pm 31$ fs, $\tau\left(D^{0}\right)=412.7 \pm 3.2 \mathrm{fs}, \tau\left(D_{s}^{+}\right)=477 \pm 12$ fs.

In Table I the amplitudes $T, C, E$, and $A$ were described above; in Table II the amplitudes are labelled with subscripts which denote the meson containing the spectator quark: $P$ for pseudoscalar, $V$ for vector [23].

We omit contributions of disconnected diagrams [24, 25] in which $\eta$ and $\eta^{\prime}$ exchange no quark lines with the rest of the diagram, and couple through their SU(3)-singlet components. Such diagrams are apparently important for the understanding of the decays $B \rightarrow K \eta^{\prime}$ [26]. They will be discussed in Sec. VII. 
Table I: Rates and invariant amplitudes for Cabibbo-favored decays of charmed mesons to two pseudoscalar mesons.

\begin{tabular}{rccccc}
\hline \hline Decay & $\begin{array}{c}M \\
(\mathrm{GeV})\end{array}$ & $\begin{array}{c}\text { Rate } \\
\left(10^{10} \mathrm{~s}^{-1}\right)\end{array}$ & $\begin{array}{c}p^{*} \\
(\mathrm{MeV})\end{array}$ & $\begin{array}{c}|\mathcal{A}| \\
\left(10^{-6} \mathrm{GeV}\right)\end{array}$ & Representation \\
\hline \hline$D^{+} \rightarrow K^{0} \pi^{+}$ & 1.8693 & $2.75 \pm 0.25$ & 862 & $1.36 \pm 0.06$ & $C+T$ \\
\hline$D^{0} \rightarrow K^{-} \pi^{+}$ & 1.8646 & $9.33 \pm 0.23$ & 861 & $2.50 \pm 0.03$ & $T+E$ \\
$\rightarrow \bar{K}^{0} \pi^{0}$ & & $5.14 \pm 0.51$ & 860 & $1.85 \pm 0.09$ & $(C-E) / \sqrt{2}$ \\
$\rightarrow \bar{K}^{0} \eta$ & & $1.72 \pm 0.24$ & 772 & $1.13 \pm 0.08$ & $C / \sqrt{3}$ \\
$\rightarrow \bar{K}^{0} \eta^{\prime}$ & & $4.17 \pm 0.63$ & 565 & $2.06 \pm 0.16$ & $-(C+3 E) / \sqrt{6}$ \\
\hline$D_{s}^{+} \rightarrow \bar{K}^{0} K^{+}$ & 1.9685 & $7.54 \pm 3.20$ & 850 & $2.38 \pm 0.36$ & $C+A$ \\
$\rightarrow \pi^{+} \eta$ & & $3.63 \pm 0.99$ & 902 & $1.61 \pm 0.22$ & $(T-2 A) / \sqrt{3}$ \\
$\rightarrow \pi^{+} \eta^{\prime}$ & & $7.78 \pm 2.15$ & 743 & $2.59 \pm 0.36$ & {$[2(T+A)] / \sqrt{6}$} \\
\hline \hline
\end{tabular}

Table II: Rates and invariant amplitudes for Cabibbo-favored decays of charmed mesons to one pseudoscalar and one vector meson.

\begin{tabular}{rccccc}
\hline \hline Decay & $\begin{array}{c}M \\
(\mathrm{GeV})\end{array}$ & $\begin{array}{c}\text { Rate } \\
\left(10^{10} \mathrm{~s}^{-1}\right)\end{array}$ & $\begin{array}{c}p^{*} \\
(\mathrm{MeV})\end{array}$ & $\begin{array}{c}\left|\mathcal{A}^{\prime}\right| \\
\left(10^{-6}\right)\end{array}$ & Representation \\
\hline \hline$D^{+} \rightarrow K^{* 0} \pi^{+}$ & 1.8693 & $1.81 \pm 0.18$ & 712 & $1.70 \pm 0.09$ & $T_{V}+C_{P}$ \\
\hline$D^{+} \rightarrow \bar{K}^{0} \rho^{+}$ & & $6.28 \pm 2.38$ & 680 & $3.40 \pm 0.64$ & $T_{P}+C_{V}$ \\
\hline$D^{0} \rightarrow K^{*-} \pi^{+}$ & 1.8646 & $12.4 \pm 0.97$ & 711 & $4.45 \pm 0.17$ & $T_{V}+E_{P}$ \\
$\rightarrow K^{-} \rho^{+}$ & & $26.2 \pm 2.4$ & 678 & $6.95 \pm 0.32$ & $T_{P}+E_{V}$ \\
$\rightarrow \bar{K}^{* 0} \pi^{0}$ & & $7.75 \pm 0.97$ & 709 & $3.54 \pm 0.22$ & $\left(C_{P}-E_{P}\right) / \sqrt{2}$ \\
$\rightarrow \bar{K}^{0}$ & $2.93 \pm 0.41$ & 676 & $2.34 \pm 0.16$ & $\left(C_{V}-E_{V}\right) / \sqrt{2}$ \\
$\rightarrow \bar{K}^{* 0} \eta$ & & $4.60 \pm 1.21$ & 580 & $3.68 \pm 0.48$ & $\left(C_{P}+E_{P}-E_{V}\right) / \sqrt{3}$ \\
$\rightarrow \bar{K}^{* 0} \eta^{\prime}$ & & $<0.27$ & 99 & $<13$ & $-\left(C_{P}+E_{P}+2 E_{V}\right) / \sqrt{6}$ \\
$\rightarrow \bar{K}^{0} \omega$ & & $5.09 \pm 0.97$ & 670 & $3.12 \pm 0.30$ & $-\left(C_{V}+E_{V}\right) / \sqrt{2}$ \\
$\rightarrow \bar{K}^{0} \phi$ & & $2.08 \pm 0.24$ & 520 & $2.92 \pm 0.17$ & $-E_{P}$ \\
\hline$D_{s}^{+} \rightarrow K^{* 0} K^{+}$ & 1.9685 & $6.91 \pm 1.89$ & 682 & $3.74 \pm 0.51$ & $C_{P}+A_{V}$ \\
$\rightarrow \bar{K}^{0} K^{*+}$ & & $9.01 \pm 2.93$ & 683 & $4.26 \pm 0.69$ & $C_{V}+A_{P}$ \\
$\rightarrow \rho^{+} \eta$ & & $22.5 \pm 6.5$ & 727 & $6.13 \pm 0.89$ & $\left(T_{P}-A_{P}-A_{V}\right) / \sqrt{3}$ \\
$\rightarrow \rho^{+} \eta^{\prime}$ & & $21.0 \pm 6.1$ & 470 & $11.4 \pm 1.7$ & $\left.\left[2 T_{P}+A_{P}+A_{V}\right)\right] / \sqrt{6}$ \\
$\rightarrow \pi^{+} \rho^{0}$ & $<0.17$ & 827 & $<0.44$ & $\left(A_{V}-A_{P}\right) / \sqrt{2}$ \\
$\rightarrow \pi^{+} \omega$ & & $0.65 \pm 0.29$ & 822 & $0.87 \pm 0.20$ & $\left(A_{V}+A_{P}\right) / \sqrt{2}$ \\
$\rightarrow \pi^{+} \phi$ & $7.54 \pm 1.89$ & 712 & $3.66 \pm 0.46$ & $T_{V}$ \\
\hline \hline
\end{tabular}




\section{REDUCED AMPLITUDES: MAGNITUDES AND PHASES}

Rather than performing a $\chi^{2}$ fit, we show what information each amplitude provides, and build up a graphical construction of the reduced amplitudes $T, C$, E, etc., which exhibits their relative phases and magnitudes. In this way it is easier to spot regularities. We are not greatly concerned with errors on the fitted quantities in the present work, since most decays are well-fitted while $D_{s} \rightarrow \rho^{+} \eta^{\prime}$ is notably poorly reproduced, as has been noted elsewhere [24, 25, 27].

\section{A. PP decays}

In the limit in which disconnected graphs do not contribute to $D \rightarrow P P$ decays, we find that $|C|$ is given by the $D^{0} \rightarrow \bar{K}^{0}$ amplitude:

$$
|C|=\sqrt{3}\left|\mathcal{A}\left(\bar{K}^{0} \eta\right)\right|=(1.96 \pm 0.14) \times 10^{-6} \mathrm{GeV},
$$

while by taking appropriate combinations of squares of amplitudes for $D^{0} \rightarrow \bar{K}^{0} \pi^{0}$, $D^{0} \rightarrow \bar{K}^{0} \eta$, and $D^{0} \rightarrow \bar{K}^{0} \eta^{\prime}$ we can eliminate the $C-E$ interference term to obtain

$$
|E|=\left\{\frac{1}{2}\left[\left|\mathcal{A}\left(\bar{K}^{0} \pi^{0}\right)\right|^{2}+\left|\mathcal{A}\left(\bar{K}^{0} \eta^{\prime}\right)\right|^{2}\right]-\left|\mathcal{A}\left(\bar{K}^{0} \eta\right)\right|^{2}\right\}^{1 / 2}=(1.60 \pm 0.13) \times 10^{-6} \mathrm{GeV} .
$$

The relative phase between $C$ and $E$ is given by

$$
\cos \delta_{C E}=\left[\frac{1}{4}\left|\mathcal{A}\left(\bar{K}^{0} \eta^{\prime}\right)\right|^{2}+\left|\mathcal{A}\left(\bar{K}^{0} \eta\right)\right|^{2}-\frac{3}{4}\left[\left|\mathcal{A}\left(\bar{K}^{0} \pi^{0}\right)\right|^{2}\right] /|C||E|=-0.07 \pm 0.11\right.
$$

or $\delta_{C E}=(94 \pm 6)^{\circ}$. The amplitudes $C$ and $E$ are depicted in Fig. 1, along with a line $C-E=\sqrt{2} \mathcal{A}\left(D^{0} \rightarrow \bar{K}^{0} \pi^{0}\right)$.

Next we use the rates for $D^{+} \rightarrow \bar{K}^{0} \pi^{+}$and $D^{0} \rightarrow K^{-} \pi^{+}$to specify the magnitudes of $T+C$ and $T+E$, respectively. Lines corresponding to these amplitudes form a triangle together with $C-E$ in the complex plane, a consquence of the isospin relation $\mathcal{A}\left(K^{-} \pi^{+}\right)+\sqrt{2} \mathcal{A}\left(\bar{K}^{0} \pi^{0}\right)=\mathcal{A}\left(\bar{K}^{0} \pi^{+}\right)$. This triangle can have either of two orientations corresponding to reflection about the line corresponding to $C-E$. These orientations correspond to different values of $T$. In Fig. 1 we denote the favored orientation by solid lines. With this choice, the value of $|T| \simeq 2.7 \times 10^{-6} \mathrm{GeV}$ is closer to that predicted by factorization $|T| \simeq 2.0 \times 10^{-6} \mathrm{GeV}$ (Sec. V), and $|C|<|T|$ as one might expect for a color-suppressed amplitude. The other choice, shown by the dashed lines and the primed amplitude $T^{\prime}$, has $|C|>\left|T^{\prime}\right| \simeq 1.1 \times 10^{-6} \mathrm{GeV}$. The determination of $T$ and $T^{\prime}$ numerically is a simple matter of solving a pair of simultaneous quadratic equations; the central values are shown in Table III. In what follows we shall consider only the large- $|T|$ solution.

To test the above construction for consistency (particularly for the validity of the assumption that no additional amplitudes are needed to describe decays involving $\eta$ and $\eta^{\prime}$ ) we consider the $D_{s}$ decays listed in Table I. We can extract the magnitude of the "annihilation" amplitude $|A|$ from the sum

$$
\left|\mathcal{A}\left(\pi^{+} \eta\right)\right|^{2}+\left|\mathcal{A}\left(\pi^{+} \eta^{\prime}\right)\right|^{2}=|T|^{2}+2|A|^{2}
$$




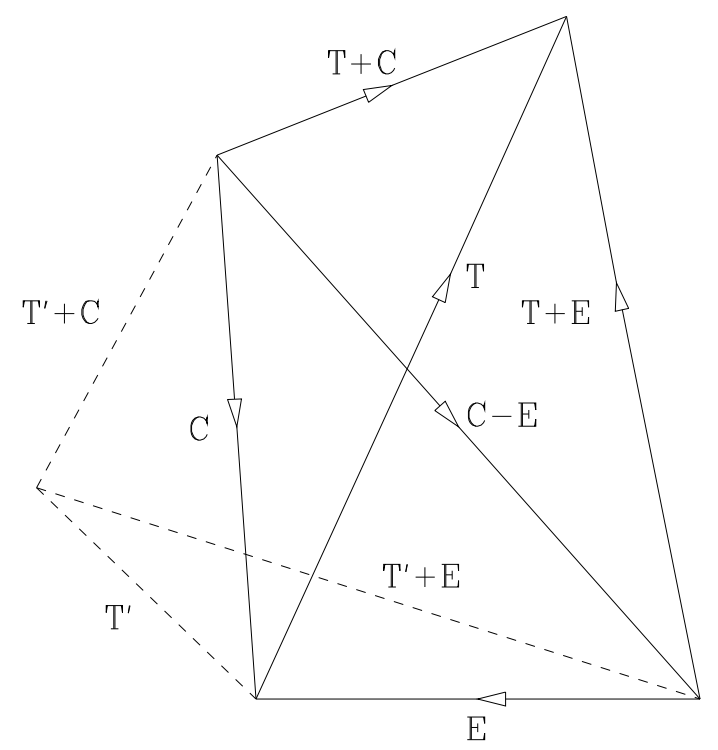

Figure 1: Amplitudes $T, C, E$ contributing to $D \rightarrow P P$ decays. $T^{\prime}$ denotes an alternate (disfavored) solution.

Table III: Central values for the amplitudes $T$ (large solution) or $T^{\prime}$ (small solution) based on the decays $D \rightarrow\left(\bar{K} \pi, \bar{K} \eta, \bar{K} \eta^{\prime}\right)$.

\begin{tabular}{cccc}
\hline \hline Solution & $|T|\left(10^{-6} \mathrm{GeV}\right)$ & $\left|\delta_{E T}\right|$ & $\left|\delta_{C T}\right|$ \\
\hline Large $|T|$ & 2.69 & $114^{\circ}$ & $152^{\circ}$ \\
Small $|T|$ & 1.08 & $44^{\circ}$ & $138^{\circ}$ \\
\hline \hline
\end{tabular}

and the value of $|T|^{2}$ from Table III to obtain $|A|=1.01 \times 10^{-6} \mathrm{GeV}$.

Using the magnitudes of $T-2 A$ and $T+A$ implied by Table I, we then may solve for the phase of $A$ and the corresponding magnitude of $C+A$. The two solutions are shown in Fig. 2 and summarized in Table IV. The solution corresponding to the unprimed amplitude $A$ in Fig. 2 agrees with the value $|C+A|=(2.38 \pm 0.36) \times 10^{-6} \mathrm{GeV}$ implied in Table I by the rate for $D_{s}^{+} \rightarrow \bar{K}^{0} K^{+}$, while that corresponding to the primed amplitude $A^{\prime}$ gives too small a value of $\left|C+A^{\prime}\right|$.

Aside from an irrelevant sign, it is interesting that the phases of $E$ and $A$ are almost identical. This could be a sign of the universal behavior of rescattering contributions conjectured in Ref. [3]. The fact that the magnitudes are not too different from one another is interesting, but we do not have a ready explanation for it at the moment.

So far we have merely shown that there is a consistent solution for the amplitudes in Cabibbo-favored decays of charmed mesons to $P P$. The one test of this consistency is the agreement of the predicted rate for $D_{s}^{+} \rightarrow \bar{K}^{0} K^{+}$when one of the discrete solutions for amplitudes is chosen. The comparison of this set of amplitudes with ones contributing to Cabibbo-favored $P V$ decays, however, suggests that the solution may have some validity. 


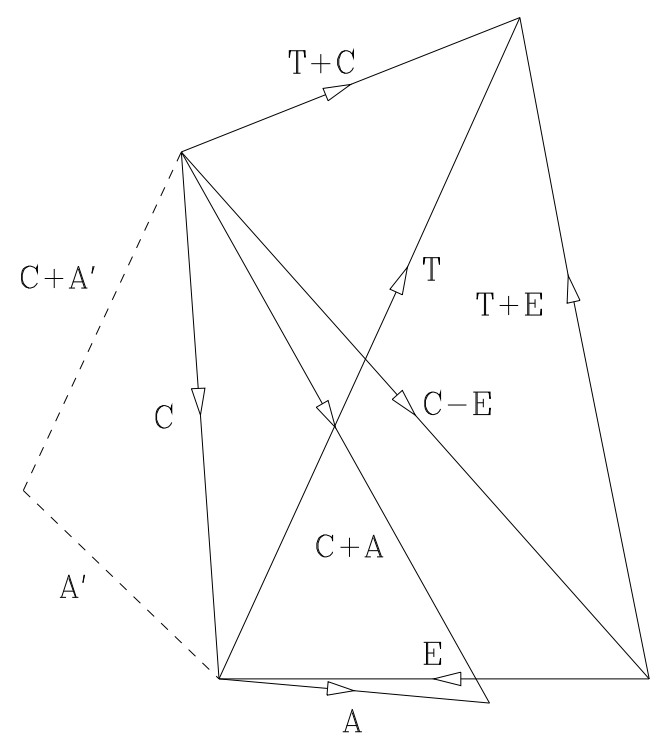

Figure 2: Amplitudes $T, C, E, A$ contributing to $D \rightarrow P P$ decays. $A^{\prime}$ denotes an alternate (disfavored) solution.

Table IV: Parameters of the solutions for $A$.

\begin{tabular}{ccc}
\hline \hline Solution & $\left|\delta_{A E}\right|$ & $|C+A|\left(10^{-6} \mathrm{GeV}\right)$ \\
\hline Favored & $175^{\circ}$ & 2.35 \\
Disfavored & $36^{\circ}$ & 1.38 \\
\hline \hline
\end{tabular}

\section{B. PV decays}

The magnitudes of $T_{V}$ and $E_{P}$ are given by the $D_{s} \rightarrow \pi^{+} \phi$ and $D^{0} \rightarrow \bar{K}^{0} \phi$ amplitudes, respectively:

$$
\begin{aligned}
& \left|T_{V}\right|=\left|\mathcal{A}^{\prime}\left(\pi^{+} \phi\right)\right|=(3.66 \pm 0.46) \times 10^{-6}, \\
& \left|E_{P}\right|=\left|\mathcal{A}^{\prime}\left(\bar{K}^{0} \phi\right)\right|=(2.92 \pm 0.17) \times 10^{-6},
\end{aligned}
$$

The relative phase of $T_{V}$ and $E_{P}$ is given by

$$
\cos \left(\delta_{E_{P}, T_{V}}\right)=\left[\left|\mathcal{A}^{\prime}\left(K^{*-} \pi^{+}\right)\right|^{2}-\left|\mathcal{A}^{\prime}\left(\pi^{+} \phi\right)\right|^{2}-\left|\mathcal{A}^{\prime}\left(\bar{K}^{0} \phi\right)\right|^{2}\right] / 2\left|T_{V}\right|\left|E_{P}\right|=-0.10 \pm 0.18,
$$

or $\delta_{E_{P}, T_{V}}=(96 \pm 10)^{\circ}$. The amplitudes $T_{V}$ and $E_{P}$ are shown in Fig. 3, along with the line $T_{V}+E_{P}=\mathcal{A}^{\prime}\left(\left(K^{*-} \pi^{+}\right)\right.$. We neglect disconnected graphs involving $\omega$ and $\phi$ since, in contrast to $\eta$ and $\eta^{\prime}$, these seem to satisfy the Okubo-Zweig-Iizuka (OZI) rule 28] well in a wide variety of processes. 


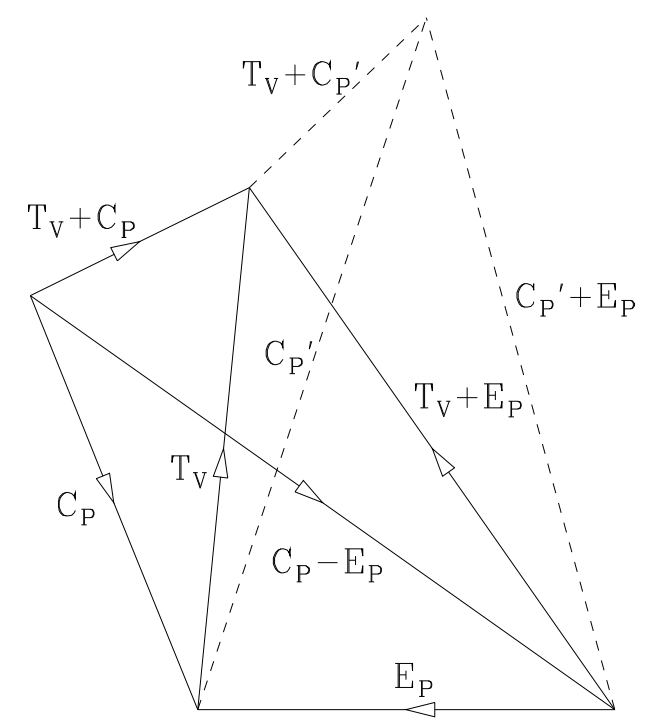

Figure 3: Amplitudes $T_{V}, C_{P}, E_{P}$ contributing to $D \rightarrow P V$ decays. $C_{P}^{\prime}$ denotes an alternate (disfavored) solution.

Table V: Central values for the amplitudes $C_{P}$ (favored solution) or $C_{P}^{\prime}$ (disfavored solution) based on the decays $D \rightarrow\left(\bar{K}^{*} \pi, \bar{K}^{0} \phi\right)$ and $D_{s}^{+} \rightarrow \pi^{+} \phi$.

\begin{tabular}{cccc}
\hline \hline Solution & $\left|C_{P}\right|\left(10^{-6}\right)$ & $\left|\delta_{C_{P}, E_{P}}\right|$ & $\left|\delta_{C_{P}, T_{V}}\right|$ \\
\hline Favored & 3.11 & $112^{\circ}$ & $152^{\circ}$ \\
Disfavored & 5.08 & $72^{\circ}$ & $168^{\circ}$ \\
\hline \hline
\end{tabular}

The rates for $D^{+} \rightarrow \bar{K}^{* 0} \pi^{+}$and $D^{0} \rightarrow \bar{K}^{* 0} \pi^{0}$ then specify the magnitudes of $T_{V}+$ $C_{P}$ and $C_{P}-E_{P}$, leading to two possible solutions. In one solution, $\left|C_{P}\right|<\left|T_{V}\right|$, as expected for a color-suppressed amplitude, while in the other, $\left|C_{P}^{\prime}\right|>\left|T_{V}\right|$, which we regard as disfavored. The favored solution is denoted by solid lines in Fig. 3, while the disfavored solution is denoted by dashed lines. The two solutions are compared in Table $\mathrm{V}$. Note that for the favored solution, the relative phase of the color-suppressed and tree amplitudes is exactly the same $\left(152^{\circ}\right)$ as in the $D \rightarrow P P$ case analyzed above.

A further set of amplitudes may be specified if one is willing to assume that $E_{V}=$ $-E_{P}$. This assumption is reasonable if the $E$ amplitude is dominated by a quarkantiquark intermediate state, since it is then a consequence of charge-conjugation invariance. It is equivalent to the assumption made by Lipkin [29] in discussing the relative penguin contributions to $B \rightarrow K^{*} \eta$ and $B \rightarrow K^{*} \eta^{\prime}$ decays. One can then construct a set of amplitudes based on the decays $D \rightarrow(\bar{K} \rho, \bar{K} \omega, \bar{K} \phi)$.

One first notes that the $D \rightarrow \bar{K} \rho$ amplitudes barely satisfy the isospin triangle relation $\mathcal{A}^{\prime}\left(\bar{K}^{0} \rho^{+}\right)=\mathcal{A}^{\prime}\left(K^{-} \rho^{+}\right)+\sqrt{2} \mathcal{A}^{\prime}\left(\bar{K}^{0} \rho^{0}\right)$. We perform a $\chi^{2}$ fit in which the 


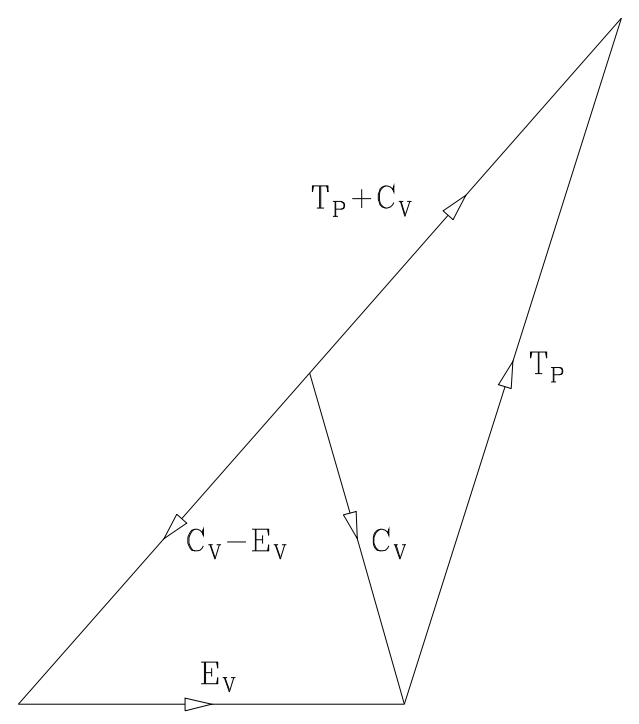

Figure 4: Amplitudes $T_{P}, C_{V}, E_{V}$ contributing to $D \rightarrow \bar{K}(\rho, \omega, \phi)$ decays.

amplitudes are relatively real and find the best fit when

$$
\left|\mathcal{A}^{\prime}\left(\bar{K}^{0} \rho^{+}\right)\right|=3.57 \times 10^{-6}, \quad\left|\mathcal{A}^{\prime}\left(K^{-} \rho^{+}\right)\right|=6.90 \times 10^{-6}, \quad\left|\mathcal{A}^{\prime}\left(\bar{K}^{0} \rho^{0}\right)\right|=3.33 \times 10^{-6},
$$

which correspond to minor displacements from the central values in Table II. We consequently shift those central values while maintaining the experimental errors.

By combining the squared amplitudes for $D^{0} \rightarrow K^{0} \rho^{0}$ and $D^{0} \rightarrow \rho^{0} \omega$ we then obtain $\left|E_{V}\right|^{2}+\left|C_{V}\right|^{2}=(15.28 \pm 2.01) \times 10^{-12}$, and recalling our assumption that $E_{V}=-E_{P}$, with $\left|E_{P}\right|^{2}=(8.51 \pm 0.98) \times 10^{-12}$, we have

$$
\left|C_{V}\right|=2.60 \pm 0.43
$$

Furthermore,

$$
\cos \delta_{C_{V}, E_{V}}=\left[\left|\mathcal{A}^{\prime}\left(\bar{K}^{0} \omega\right)\right|^{2}-\left|\mathcal{A}^{\prime}\left(\bar{K}^{0} \rho^{0}\right)\right|^{2}\right] / 2\left|C_{V}\right|\left|E_{V}\right|=0.28 \pm 0.14
$$

or $\delta_{C_{V}, E_{V}}=(74 \pm 8)^{\circ}$. The amplitudes $E_{V}, C_{V}$, and $C_{V}-E_{V}$ are shown in Fig. 4 .

Since the best fit to $D \rightarrow \bar{K} \rho$ decays is obtained when all three amplitudes are relatively real, we use the fact that $\operatorname{Arg}\left(T_{P}+E_{V}\right)=\operatorname{Arg}\left(E_{V}-C_{V}\right) \simeq 49^{\circ}$ to construct the amplitudes $T_{P}+E_{V}, T_{P}+C_{V}$, and $T_{P}$. The results are:

$$
\left|T_{P}\right|=5.44 \times 10^{-6}, \quad \delta_{E_{V}, T_{P}}=72^{\circ}, \quad \delta_{C_{V}, T_{P}}=148^{\circ} .
$$

The relative phase of the color-suppressed and tree amplitudes is very similar to that in the two previous constructions, as one sees from Fig. 4.

The shape of Fig. 4 is very different from that of the two previous figures. The fact that the $\bar{K} \rho$ amplitudes are in phase with one another appears to be the consequence 
of the sign flip of $E_{V}$ relative to $E_{P}$ or $E$. This would not have had such a noticeable effect were it not for the fact that, at least in our fits, the tree and color-suppressed amplitudes all possess a relative phase of about $150^{\circ}$.

One more test of the assumption $E_{V}=-E_{P}$ is passed at about the $1 \sigma$ level. The amplitude for $D^{0} \rightarrow \bar{K}^{* 0} \eta$ is predicted to be $\mathcal{A}^{\prime}\left(\bar{K}^{* 0} \eta\right)=\left(C_{P}+2 E_{P}\right) / \sqrt{3}$ and, as a consequence of the amplitudes determined above, is predicted to have magnitude $\left|\mathcal{A}^{\prime}\left(\bar{K}^{* 0} \eta\right)\right|=3.17 \times 10^{-6}$. This is in satisfactory agreement with the experimental value $\left|\mathcal{A}^{\prime}\left(\bar{K}^{* 0} \eta\right)\right|=(3.68 \pm 0.48) \times 10^{-6}$. The amplitude for $D^{0} \rightarrow \bar{K}^{* 0} \eta^{\prime}$ is predicted to be $\mathcal{A}^{\prime}\left(\bar{K}^{* 0} \eta\right)=-\left(C_{P}-E_{P}\right) / \sqrt{6}=-\mathcal{A}^{\prime}\left(\bar{K}^{* 0} \pi^{0}\right) / \sqrt{3}$ with magnitude $\left|\mathcal{A}^{\prime}\left(\bar{K}^{* 0} \eta\right)\right|=$ $(2.04 \pm 0.13) \times 10^{-6}$, much smaller than the current experimental upper bound.

\section{Comments on final-state interactions}

The conclusion of the fits to Cabibbo-favored $D \rightarrow P P$ and $D \rightarrow P V$ amplitudes is that final-state interactions (parametrized by large $E$ and $A$ contributions) are important in all final states, including the $D \rightarrow \bar{K} \rho$ decays where the amplitudes for the three charge states are all in phase with one another. The presence of large final-state phases in the $\bar{K} \rho$ case is masked by the cancellation of contributions between the "exchange" amplitude and the "color-suppressed" amplitude. This cancellation arises in $\bar{K} \rho$ decays and not in $\bar{K}^{*} \pi$ decays as a result of a sign flip in the "exchange" amplitude contribution, which is just due to the charge-conjugation invariance of the strong coupling.

\section{FACTORIZATION COMPARISONS}

We compare the values of $T, T_{V}$, and $T_{P}$ obtained above with values extracted using the factorization assumption 30, 31, 32, 33 and the spectra $d \Gamma\left(D \rightarrow \bar{K}^{(*)} \ell^{+} \nu_{\ell}\right) / d q^{2}$, where $q=p_{\ell}+p_{\nu_{\ell}}$. For simplicity we use the limit of heavy-quark effective theory and expressions derived in Ref. [33]. No QCD corrections will be applied. We shall neglect the pion mass.

\section{A. $\bar{K} \pi$ decays}

We use the relation [32]

$$
\frac{\Gamma\left(D \rightarrow \bar{K} \pi^{+}\right)_{T}}{d \Gamma\left(D \rightarrow \bar{K} \ell^{+} \nu_{\ell}\right) /\left.d q^{2}\right|_{q^{2}=m_{\pi}^{2}}}=6 \pi^{2} f_{\pi}^{2}\left|V_{u d}\right|^{2}=0.98 \mathrm{GeV}^{2}
$$

where the subscript denotes the contribution of the $T$ amplitude to the $\bar{K} \pi^{+}$decay, excluding $C$ in $\bar{K}^{0} \pi^{+}$or $E$ in $K^{-} \pi^{+}$(see Table I). Here $f_{\pi}=132 \mathrm{MeV}$. A recent spectrum for $D^{0} \rightarrow K^{-} \mu^{+} \nu_{\mu}$ has been published by the Fermilab E687 Collaboration [34]. Reading from their graph, we estimate

$$
\left.\frac{1}{\Gamma\left(D^{0} \rightarrow K^{-} \mu^{+} \nu_{\mu}\right)} \frac{d \Gamma\left(D^{0} \rightarrow K^{-} \mu^{+} \nu_{\mu}\right)}{d q^{2}}\right|_{q^{2}=m_{\pi}^{2}}=0.76 \pm 0.09 \mathrm{GeV}^{-2}
$$


Furthermore, E687 quotes

$$
\frac{\Gamma\left(D^{0} \rightarrow K^{-} \mu^{+} \nu_{\mu}\right)}{\Gamma\left(D^{0} \rightarrow K^{-} \pi^{+}\right)}=0.852 \pm 0.034 \pm 0.028
$$

Putting these pieces together, we predict

$$
\frac{\Gamma\left(D \rightarrow \bar{K} \pi^{+}\right)_{T}}{\Gamma\left(D^{0} \rightarrow K^{-} \pi^{+}\right)}=0.63 \pm 0.08
$$

or $\Gamma\left(D \rightarrow \bar{K} \pi^{+}\right)_{T}=(5.9 \pm 0.8) \times 10^{10} \mathrm{~s}^{-1}$, entailing $|T|=(1.99 \pm 0.13) \times 10^{-6} \mathrm{GeV}$. This is to be compared with the result $|T|=2.69 \times 10^{-6} \mathrm{GeV}$ obtained in the fit of Section IV: $\left|T_{\text {fit }} / T_{\text {fact }}\right| \simeq 1.35$. This is well within expectations of what QCD corrections might provide. One must be careful in applying such corrections in the present approach, however, since they will mix operators of the type $T, C$, and $E$. Our description is purely a long-distance one. A more complete treatment would probably involve a hybrid between short- and long-distance effects.

\section{B. $\bar{K}^{*} \pi$ decays}

In the heavy-quark limit, one expects $\Gamma\left(D \rightarrow \bar{K}^{*} \pi^{+}\right)_{T}=\Gamma\left(D \rightarrow \bar{K} \pi^{+}\right)_{T}$ and near $q^{2}=0 d \Gamma\left(D \rightarrow \bar{K}^{*} \ell^{+} \nu_{\ell}\right) / d q^{2}=d \Gamma\left(D \rightarrow \bar{K} \ell^{+} \nu_{\ell}\right) / d q^{2}$. In this limit the $\bar{K}^{*}$ in the semileptonic process is longitudinally polarized. Here we have used Eqs. (9), (11), and (14) of Ref. [33]. Thus we predict $\Gamma\left(D \rightarrow \bar{K}^{*} \pi^{+}\right)_{T}=(5.9 \pm 0.8) \times 10^{10} \mathrm{~s}^{-1}$, or, with the kinematic factors of Table I, $\left|T_{V}\right|=(3.08 \pm 0.21) \times 10^{-6}$. Recall that $\left|T_{V \text {,fit }}\right|=$ $(3.66 \pm 0.46) \times 10^{-6}$, where the error can be easily assigned since this quantity is obtained from the decay $D_{s} \rightarrow \pi^{+} \phi$. Then $\left|T_{V, \text { fit }} / T_{V \text {,fact }}\right|=1.19 \pm 0.17$.

\section{C. $\bar{K} \rho$ decays}

A similar approach to $D \rightarrow \bar{K} \rho$ decays utilizes the semileptonic spectrum of Fermilab E687 [34] with

$$
\left.\frac{1}{\Gamma\left(D^{0} \rightarrow K^{-} \mu^{+} \nu_{\mu}\right)} \frac{d \Gamma\left(D^{0} \rightarrow K^{-} \mu^{+} \nu_{\mu}\right)}{d q^{2}}\right|_{q^{2}=m_{\rho}^{2}}=0.74 \pm 0.09 \mathrm{GeV}^{-2}
$$

read from the graph, and $f_{\pi} \rightarrow f_{\rho} \simeq \sqrt{2} f_{\pi}$ 33. in Eq. (13), with the result $\Gamma(D \rightarrow$ $\bar{K} \rho)_{T}=(12.1 \pm 1.6) \times 10^{10} \mathrm{~s}^{-1}$, implying $\left|T_{P}\right|=(4.7 \pm 0.3) \times 10^{-6}$. The fit in Section IV gave $\left|T_{P}\right|=5.44 \times 10^{-6}$, so here we have $\left|T_{P, \text { fit }} / T_{P, \text { fact }}\right| \simeq 1.15$.

\section{Summary of factorization results}

We compare the results for $|T|,\left|T_{V}\right|$, and $\left|T_{P}\right|$ obtained from the fits of Sec. IV and those obtained via factorization in Table VI. All told, the agreement with factorization for the "tree" amplitudes in $D \rightarrow \bar{K} \pi, \bar{K}^{*} \pi, \bar{K} \rho$ decays is satisfactory. 
Table VI: Comparison of results for "tree" amplitudes obtained from fits to data and using factorization.

\begin{tabular}{cccc}
\hline \hline Method & $\begin{array}{c}|T| \\
\left(10^{-6} \mathrm{GeV}\right)\end{array}$ & $\begin{array}{c}\left|T_{V}\right| \\
\left(10^{-6}\right)\end{array}$ & $\begin{array}{c}\left|T_{P}\right| \\
\left(10^{-6}\right)\end{array}$ \\
\hline Fit & $2.69^{a}$ & $3.66 \pm 0.46$ & $5.44^{a}$ \\
Fact. & $1.99 \pm 0.13$ & $3.08 \pm 0.21$ & $4.7 \pm 0.3$ \\
Fit/Fact. & $1.35^{a}$ & $1.19 \pm 1.17$ & $1.15^{a}$ \\
\hline \hline
\end{tabular}

${ }^{a}$ Central value only. Error on fitted amplitude was not determined.

\section{RESONANT INTERPRETATIONS}

We have found two relative phases in the present sets of fits: those between $C$ and $T$, and those between $E$ and $T$ (or $C$ ) amplitudes. A resonant interpretation of the $C-T$ relative phases is not possible; we ascribe these phases rather to rescattering, most likely from the $T$ channel to the $C$ channel rather than vice versa in view of the color-suppression of the weak amplitude for the latter. Thus, it will make most sense to examine the relative phase between $E$ (or $A$ ) and $T$ amplitudes in terms of contributions of possible direct-channel resonances.

\section{A. PP decays}

A resonance contributing to the $E$ amplitude in $D \rightarrow \bar{K} \pi$ decays must have spinparity $J^{P}=0^{+}$. Such a resonance has been seen by the LASS Collaboration [35] with a mass of $M=1945 \pm 10 \pm 20 \mathrm{MeV} / c^{2}$ and a width of $\Gamma=201 \pm 34 \pm 79 \mathrm{MeV}$. [A reanalysis [36] in a $T$-matrix formalism quotes $M=1820 \pm 40 \mathrm{MeV} / c^{2}$ and $\Gamma=250 \pm 100 \mathrm{MeV}$.]

In Fig. 1 and Table III we found the relative $T-E$ phase to be $114^{\circ}$. This would indicate that $M_{D}$ was not far from a Breit-Wigner peak. If we regard the $E$ amplitude as "fed" by rescattering from the elastic $K^{-} \pi^{+}$channel, we should take $T$ to be real and positive and $E$ to have a positive imaginary part, in which case we should parametrize the propagator for a resonance with mass $M_{R}$ and width $\Gamma_{R}$ as

$$
D(M)=\frac{1}{M_{R}-M-i \Gamma_{R} / 2}=\frac{M_{R}-M+i \Gamma_{R} / 2}{\left(M-M_{R}\right)^{2}+\left(\Gamma_{R} / 2\right)^{2}},
$$

Then we would expect resonance dominance to give $\Gamma_{R} /\left[2\left(M_{R}-M_{D}\right)\right]=\tan ^{-1} \pm 114^{\circ}=$ -2.3. This supports the claim [36] that $M_{R}<M_{D}$ and is compatible with the resonant parameters found in that analysis.

The $A$ amplitude seems to have a phase very close to that of $E$. No suitable $I=1$ resonance near $M_{D_{s}}$ with $J^{P}=0^{+}$appears in the most recent compilation [20].

\section{B. PV decays}

A resonance contributing to $D \rightarrow \bar{K}^{*} \pi$ or $D \rightarrow \bar{K} \rho$ decays must have $J^{P}=0^{-}$. Normally one would expect such a resonance to have equal and opposite couplings to 
$K^{*-} \pi^{+}$and $K^{-} \rho^{+}$channels, by charge-conjugation invariance. (In SU(3) language, one expects $F$-type coupling of the resonance octet to the two final octets.)

The sign of the resonant contribution is less obvious in this case since we expect rescattering to be fed by both the $K^{*-} \pi^{+}$and $K^{-} \rho^{+}$channels. We use the result $\left|T_{P}\right|>\left|T_{V}\right|$, found both in our fits and in a factorization calculation (cf. Table VI) to argue that the dominant channel from which rescattering occurs is $K^{-} \rho^{+}$. In that case it is the $\bar{K} \rho$ channel for which the sign in Eq. (18) applies.

In Sec. IV B and Fig. 4, we found $\left|\delta_{E_{V}, T_{P}}\right|=72^{\circ}$ for the $\bar{K} \rho$ channels. Since we expect $E_{P}=-E_{V}$ and no relative phase between $T_{P}$ and $T_{V}$, we then predict $\left|\Delta_{E_{P}, T_{V}}\right|=$ $\pi-\left|\delta_{E_{V}, T_{P}}\right|=108^{\circ}$, which is marginally consistent with the value $\left|\delta_{E_{V}, T_{P}}\right|=(96 \pm 10)^{\circ}$ found in Sec. IV B.

One then expects $\Gamma_{R} /\left[2\left(M_{R}-M_{D}\right)\right]=\tan 72^{\circ}=3.1$, or $M_{R}>M_{D}$. A $0^{-}$resonance is seen [37] in the vicinity of $M_{D}$, but it is around $1830 \mathrm{MeV} / c^{2}$ and only its decay to $K^{-} \phi$ has been reported. If a resonant interpretation of the amplitudes $E_{P}$ and $E_{V}$ is correct, and these amplitudes are generated mainly by rescattering from the dominant $K^{-} \rho^{+}$process, we expect there to exist a $0^{-}$resonance slightly above $M_{D}$, whose width should be about 6 times the $M_{R}-M_{D}$ difference, decaying to $\bar{K}^{*} \pi$ and $\bar{K} \rho$, with equal partial widths aside from small phase space corrections.

\section{AMPLITUDES NOT FITTED}

A group of amplitudes in Table II containing the contributions of $A_{P}$ and $A_{V}$ has not been fitted. As has been noted in Refs. [24], 25], and [27], the decay $D_{s} \rightarrow \rho^{+} \eta^{\prime}$ cannot be fitted without the introduction of additional contributions from disconnected diagrams involving the flavor-SU(3)-singlet component of the $\eta^{\prime}$, which will also affect $\eta$ production to a small degree. Such a component was anticipated to be important in the decays $B \rightarrow K \eta^{\prime}$ [19], as has been borne out experimentally [38] and widely discussed theoretically [39]. As we have seen above, this component was not needed to fit any of the other decays involving $\eta$ and $\eta^{\prime}$, but its possible presence could cast some doubt on the conclusions regarding the amplitude $A$ in $P P$ decays, as well as the parameters we have determined in Figs. 3 and 4 and Table V. Let us first recapitulate the tests for these parameters presented earlier.

The value of $A$ determined in Sec. IV was found to be consistent with the decay $D_{s} \rightarrow \bar{K}^{0} K^{+}$. The parameters of Fig. 3 and Table V were found to be consistent with the rate for $D^{0} \rightarrow \bar{K}^{* 0} \eta$ and with factorization. (We do not count the prediction for the very small rate for $D^{0} \rightarrow \bar{K}^{* 0} \eta^{\prime}$ as much of a test since it relies mainly on the very small available phase space.) The parameters of Fig. 4 were consistent with factorization. It is possible that by appeal to Cabibbo-forbidden decays and liberal use of (possibly broken) flavor-SU(3) one could glean additional information, but that is beyond the scope of the present paper.

To see the nature of the problem, we compare

$$
\left|\mathcal{A}^{\prime}\left(\rho^{+} \eta\right)\right|^{2}+\left|\mathcal{A}^{\prime}\left(\rho^{+} \eta^{\prime}\right)\right|^{2}=\left|T_{P}\right|^{2}+\frac{1}{2}\left[\left|A_{P}\right|^{2}+\left|A_{V}\right|^{2}\right]=(167 \pm 39) \times 10^{-12}
$$


with

$$
0.42 \times 10^{-12}<\left|\mathcal{A}^{\prime}\left(\pi^{+} \rho^{0}\right)\right|^{2}+\left|\mathcal{A}^{\prime}\left(\pi^{+} \omega\right)\right|^{2}=\left|A_{P}\right|^{2}+\left|A_{V}\right|^{2}<1.34 \times 10^{-12}
$$

[using the upper bound on $\Gamma\left(\pi^{+} \rho^{0}\right)$ and the $1 \sigma$ bounds on $\Gamma\left(\pi^{+} \omega\right)$ ] to conclude that $\left|T_{P}\right|>11.3 \times 10^{-6}$, to be compared with the fitted value of $5.4 \times 10^{-6}$. At the same time, if we omit the $\rho^{+} \eta^{\prime}$ decay from the fit, we find no difficulty in constructing a set of amplitudes fitting the rates for $D_{s} \rightarrow\left(\bar{K}^{* 0} K^{+}, \bar{K}^{0} K^{*+}, \pi^{+} \omega\right)$ and the upper limit for $D_{s} \rightarrow \pi^{+} \rho^{0}$, though the absence of a measurement for this last process prevents us from specifying the parameters. Since we already have information on $C_{P}$ and $C_{V}$ (including their relative phase, which is small), we need both magnitudes and phases for $A_{P}$ and $A_{V}$. Without four measured decay rates or some additional assumption, such information is unavailable.

In view of the upper bound (20) on the contribution of the annihilation amplitudes, one might have expected the $\rho^{+} \eta$ and $\rho^{+} \eta^{\prime}$ rates to be dominated by the $T_{P}$ amplitude. However, comparing the CLEO measurement 21]

$$
\frac{\mathcal{B}\left(D_{s} \rightarrow \rho^{+} \eta^{\prime}\right)}{\mathcal{B}\left(D_{s} \rightarrow \rho^{+} \eta\right)}=0.93 \pm 0.19
$$

with the corresponding ratio of semileptonic branching ratios 40

$$
\frac{\mathcal{B}\left(D_{s} \rightarrow e^{+} \nu_{e} \eta^{\prime}\right)}{\mathcal{B}\left(D_{s} \rightarrow e^{+} \nu_{e} \eta\right)}=0.35 \pm 0.09 \pm 0.07,
$$

there must be an additional contribution which is particularly important for the decay $\rho^{+} \eta^{\prime}$. Such a contribution would be provided by a disconnected quark diagram.

One might be tempted to ascribe $A_{P}$ and $A_{V}$ to the contribution of a $q \bar{q}$ resonance. However, such an interpretation would entail the relation $A_{P}+A_{V}=0$ (since there is no $I=1 q \bar{q} 0^{+}$resonance which can couple to $\left.\pi^{+} \omega\right)$. This would run counter to the observation of $D_{s} \rightarrow \pi^{+} \omega$ and would entail very small values of both $A_{P}$ and $A_{V}$, leading to difficulty in fitting the $\bar{K}^{0} K^{*+}$ rate. It is more likely that $A_{P}$ and $A_{V}$ have a relative phase less than $\pi / 2$ with respect to each other and with respect to $C_{P}$ and $C_{V}$.

We are left with the possibility that disconnected graphs play a role in the decay $D_{s} \rightarrow \rho^{+} \eta^{\prime}$. The remaining processes seem to be described satisfactorily without such contributions 41], but some of them will be affected when they are included. In such a case, however, one cannot specify the parameters of the fits without additional assumptions.

\section{SUMMARY AND DISCUSSION}

The apparent puzzle of large relative phases between $D \rightarrow \bar{K} \pi$ and $D \rightarrow \bar{K}^{*} \pi$ amplitudes but relatively real $D \rightarrow \bar{K} \rho$ amplitudes has been explained. Amplitudes with large final-state phases are present in all three classes of decays, but their effects are masked by accidental cancellations in the $D \rightarrow \bar{K} \rho$ case. The reason that this cancellation can occur is that there are two types of amplitudes which can have phases relative to the 
"tree" process $T$ : Both the color-suppressed amplitudes $C$ and the exchange amplitudes $E$ (or annihilation amplitudes $A$ ) have such phases.

The relative phases between $C$ and $T$ amplitudes seem to be about $150^{\circ}$ in all three sets of processes. These presumably arise from a rescattering process in which the $C$ amplitudes are fed by $T$ contributions. A sign flip in the $E$ amplitude is responsible for the difference between $\bar{K}^{*} \pi$ and $\bar{K} \rho$ behavior.

The present fit implies tree amplitudes $T$ which are fairly close to those obtained from semileptonic $D$ decays and factorization, and fits data for such processes as $D_{s} \rightarrow \bar{K}^{0} K^{+}$ and $D^{0} \rightarrow \bar{K}^{* 0} \eta$ without additional parameters. In company with a number of other approaches, it fails to fit the decay $D_{s} \rightarrow \rho^{+} \eta^{\prime}$, since disconnected diagrams involving the flavor-SU(3)-singlet component of the $\eta^{\prime}$ have not been taken into account. These are expected to also play a (much smaller) role in the decay $D_{s} \rightarrow \rho^{+} \eta$, and possibly in other processes involving $\eta$ and $\eta^{\prime}$ as well.

One might be tempted to draw conclusions about final-state effects in weak decays of hadrons lighter or heavier than $D$ 's from the above results. In the case of charmed mesons one sees that the amplitudes $A$ and $E$ are of comparable magnitude to tree amplitudes $T$ and their color-suppressed versions $C$. This approximate equality is probably a way to understand why different charmed particles differ in lifetimes by factors of a few, e.g., $\tau\left(D^{+}\right) / \tau\left(D^{0}\right) \simeq 2.5$. (Short-distance discussions of these differences provide illuminating and probably complementary insights 42.) In $K \rightarrow 2 \pi$ decays one can ascribe at least part of the 20 -fold enhancement of the $I=0$ amplitude with respect to the $I=2$ amplitude to such effects. Thus, as the mass of the decaying particle increases by a factor of 3, the effects of the final-state interactions seem to decrease (in amplitude) by roughly a factor of 10 . If this trend is extrapolated to $B$ particles, one would expect final-state-interaction amplitudes to be suppressed with respect to "tree" processes by the same factor of 10. If they have large phases with respect to the tree processes they will not show up in lifetime differences at present levels of sensitivity, while in certain cases (e.g., $\Lambda_{b}$ decays) they might be in phase with tree amplitudes and could give rise to effects in the 10-20\% range, perhaps accounting for the observed ratio $\tau\left(\Lambda_{b}\right) / \tau(B) \simeq 0.8$ in contrast to less successful attempts [43, 44] based purely on short-distance arguments.

Final-state interaction effects also might lead to contributions interfering with amplitudes such as the penguin amplitude assumed to dominate $B^{+} \rightarrow \bar{K}^{0} \pi^{+}$decays. Elsewhere [16, 45, 46] we have speculated that amplitudes which involve the spectator quark (such as $E$ and $A$ ) would be suppressed relative to amplitudes not involving the spectator (such as $T$ ) in decays of mesons $M$ by a factor of $f_{M} / M_{M}$, where $f_{M}$ is the decay constant of the corresponding meson. This hierarchy does not appear to be respected in the present example of charmed particles, where we find $|E|=\mathcal{O}(|T|)$ but $f_{D} / M_{D} \simeq(200 \mathrm{MeV}) /(1.9 \mathrm{GeV}) \simeq 0.1$. If, however, final-state effects fall off roughly as $1 / M_{M}^{2}$ they will lead to values of $|E / T|$ and $|A / T|$ closer to (but still in excess of) $f_{B} / M_{B} \simeq(200 \mathrm{MeV}) /(5 \mathrm{GeV}) \simeq 0.04$.

The present description has been a purely long-distance one. It could probably be adapted to a hybrid treatment of both short- and long-distance effects, reminiscent of that taken by Ciuchini et al. [47] to describe the enhancement of "charming penguin" amplitudes in $B$ decays. Purely short-distance descriptions of the matrix elements of 
penguin operators fall short of those needed to explain a number of $B \rightarrow K \pi$ processes, particularly in such processes as $B^{+} \rightarrow K^{+} \omega$ [48] in which the spectator quark ends up in a vector meson [23]. The purely short-distance approach to final-state phases [49] involves calculations of imaginary parts at the quark level, which could well underestimate the importance of such effects even at masses as high as a few $\mathrm{GeV} / c^{2}$.

\section{ACKNOWLEDGEMENTS}

I would like to thank the Physics Department and the Theory Group at the University of Hawaii for their hospitality during part of this work, and S. Olsen, S. Pakvasa, S. F. Tuan, and H. Yamamoto for helpful conversations there. I would also like to thank H. J. Lipkin for discussions on final-state interactions. This work was supported in part by the United States Department of Energy under Contract No. DE FG02 90ER40560.

\section{References}

[1] A. B. Carter and A. I. Sanda, Phys. Rev. D 23, 1567 (1981); T. Brown, S. F. Tuan, and S. Pakvasa, Phys. Rev. Lett. 51, 1823 (1983).

[2] L. Wolfenstein, Phys. Rev. D 52, 537 (1995); M. Gronau, J. L. Rosner and D. London, CERN-TH.7407/94, EFI-94-41, 1994 (unpublished); see also Sec. VI E of M. Gronau, O. F. Hernández, D. London, and J. L. Rosner, Phys. Rev. D 52, 6356 (1995); J. F. Donoghue, E. Golowich, A. A. Petrov and J. M. Soares, Phys. Rev. Lett. 77, 2178 (1996); B. Blok and I. Halperin, Phys. Lett. B 385, 324 (1996); B. Blok, M. Gronau, and J. L. Rosner, Phys. Rev. Lett. 78, 3999 (1997); A. J. Buras, R. Fleischer, and T. Mannel, Nucl. Phys. B533, 3 (1998); J.-M. Gérard and J. Weyers, Eur. Phys. J. C 7, 1 (1999); D. Delépine, J. M. Gérard, J. Pestiau, and J. Weyers, Phys. Lett. B 429, 106 (1998); J. M. Gérard, J. Pestiau, and J. Weyers, ibid. 436, 363 (1998); D. Atwood and A. Soni, Phys. Rev. D 58, 036005 (1998); A. Falk, A. L. Kagan, Y. Nir, and A. A. Petrov, Phys. Rev. D 57 , 6843 (1998); M. Neubert, Phys. Lett. B 424, 152 (1998); M. Suzuki, Lawrence Berkelely Laboratory report LBL-42025, 1998, hep-ph/9807414 (unpublished); R. Fleischer, Phys. Lett. B 435, 221 (1998); Eur. Phys. J. C 6, 451 (1999); A. Buras and R. Fleischer, CERN report CERN-TH/98-319, hep-ph/9810260 (unpublished); M. Gronau and J. L. Rosner, Phys. Rev. D 58, 113005 (1998); A. N. Kamal, University of Alberta report ALBERTA-THY-01-99, hep-ph/9901342 (unpublished); M. Gronau and D. Pirjol, Cornell University report CLNS-99-1604, hep-ph/9902482 (unpublished); M. Suzuki and L. Wolfenstein, Lawrence Berkeley Laboratory report LBNL-43001, hep-ph/9903477 (unpublished).

[3] J. L. Rosner, Enrico Fermi Institute Report No. EFI-99-10, hep-ph/9903543, to be published in Phys. Rev. D.

[4] M. Kobayashi and T. Maskawa, Prog. Theor. Phys. 49, 652 (1973). 
[5] Mark III Collaboration, J. Adler et al., Phys. Lett. B 196, 107 (1987).

[6] A. N. Kamal and R. C. Verma, Phys. Rev. D 35, 3515 (1987), ibid. 36, 3527(E) (1987).

[7] J. L. Rosner, in Particles and Fields 3, Proceedings of the Banff Summer Institute (CAP) 1988, 14-26 August 1988, edited by A. N. Kamal and F. C. Khanna (World Scientific, Singapore, 1989), p. 395, and references therein.

[8] M. Suzuki, Phys. Rev. D 58, 111504 (1998).

[9] Fermilab E691 Collaboration, J. C. Anjos et al., Phys. Rev. D 48, 56 (1993).

[10] ARGUS Collaboration, H. Albrecht et al., Phys. Lett. B 308, 435 (1993).

[11] Fermilab E687 Collaboration, P. Frabetti et al., Phys. Lett. B 331, 217 (1994).

[12] C. Quigg, Zeit. Phys. C 4, 55 (1980).

[13] D. Zeppenfeld, Zeit. Phys. C 8, 77 (1981).

[14] M. Savage and M. Wise, Phys. Rev. D 39, 3346 (1989); ibid. 40, 3127(E) (1989).

[15] L. L. Chau et al., Phys. Rev. D 43, 2176 (1991), and references therein.

[16] M. Gronau, Oscar F. Hernández, D. London, and J. L. Rosner, Phys. Rev. D 50, 4529 (1994), ibid. 52, 6356, 6374 (1995).

[17] See, e.g., L. L. Chau, Phys. Rep. 95, 1 (1983); L. L. Chau and H. Y. Cheng, Phys. Rev. D 36, 137 (1987), ibid. 39, 2788 (1989); ibid. 42, 1837 (1990); L. L. Chau et al., Phys. Rev. D 43, 2176 (1991); L. L. Chau, H. Y. Cheng, and T. Huang, Zeit. Phys. C 53, 413 (1992); D. Lee, Phys. Lett. B 275, 469 (1992); ibid. 277, 529(E) (1992); F. Buccella et al., Phys. Rev. D 51, 3478 (1996), Phys. Lett. B 379, 246 (1996); R. C. Verma, A. N. Kamal, and M. P. Khanna, Zeit. Phys. C 65, 255 (1995), and references therein; I. Hinchliffe and T. Kaeding, Phys. Rev. D 54, 914 (1996); F. E. Close and H. J. Lipkin, Phys. Lett. B 405, 157 (1997); H.-Y. Cheng and B. Tseng, Phys. Rev. D 59, 014034 (1999).

[18] H. J. Lipkin, Phys. Rev. Lett. 44, 710 (1980); ibid. 46, 1307 (1981); in Proceedings of the Second International Conference on Hadron Spectroscopy, April 16-18, 1987, KEK, Tsukuba, Japan, edited by Y. Oyanagi, K. Takamatsu, and T. Tsuru (National Laboratory for High Energy Physics, Tsukuba, Japan, 1987), p. 363.

[19] M. Gronau and J. L. Rosner, Phys. Rev. D 53, 2516 (1996); A. S. Dighe, M. Gronau, and J. L. Rosner, Phys. Lett. B 367, 357 (1996); ibid. 377, 325(E) (1996).

[20] Particle Data Group, C. Caso et al., Eur. Phys. J. C 3, 1 (1998).

[21] CLEO Collaboration, C. P. Jessop et al., Phys. Rev. D 58, 052002 (1998). 
[22] CLEO Collaboration, G. Bonvicini et al., Cornell University report CLNS-99-1603, hep-ex/9902011 (unpublished).

[23] A. Dighe, M. Gronau, and J. L. Rosner, Phys. Rev. D 57, 1783 (1988).

[24] L. L. Chau and H.-Y. Cheng, Phys. Rev. D 39, 2788 (1989); L. L. Chau, H.-Y. Cheng, and T. Huang, Zeit. Phys. C 53, 413 (1992).

[25] P. Ball, J.-M. Frère, and M. Tytgat, Phys. Lett. B 365, 367 (1996).

[26] A. Dighe, M. Gronau, and J. L. Rosner, Phys. Rev. Lett. 79, 4333 (1997).

[27] H.-Y. Cheng and B. Tseng, Phys. Rev. D 59, 014034 (1999).

[28] S. Okubo, Phys. Rev. Lett. 5, 165-8 (1963); G. Zweig, CERN Reports 8182/CERNTH-401 and 8419/CERN-TH-412, 1974, second paper reprinted in Devlopments in the Quark Theory of Hadrons, vol. 1, edited by D. B. Lichtenberg and S. P. Rosen (Hadronic Press, Nonantum, MA, 1980), pp. 22-101; J. Iizuka, Prog. Theor. Phys. Suppl. 37-38, 21-34 (1966), and references therein.

[29] H. J. Lipkin, Phys. Lett. B 433, 117 (1998); ibid. 445, 403 (1999).

[30] M. Wirbel, B. Stech, and M. Bauer, Zeit. Phys. C 29, 637 (1985); M. Bauer, B. Stech, and M. Wirbel, Zeit. Phys. C 34, 103 (1987); M. Bauer and M. Wirbel, ibid. 42, 671 (1989).

[31] M. Neubert and B. Stech, in Heavy Flavours II, edited by A. J. Buras and M. Lindner (World Scientific, Singapore, 1998), p. 294.

[32] J. D. Bjorken, in New Developments in High-Energy Physics, Proc. IV International Workshop on High-Energy Physics, Orthodox Academy of Crete, Greece, 1-10 July 1988, edited by E. G. Floratos and A. Verganelakis, Nucl. Phys. B (Proc. Suppl.) 11, 325 (1989); D. Bortoletto and S. Stone, Phys. Rev. Lett. 65, 2951 (1990).

[33] J. L. Rosner, Phys. Rev. D 42, 3732 (1990).

[34] Fermilab E687/FOCUS Collaboration, presented by W. E. Johns in Heavy Quarks at Fixed Target, Batavia, IL, 1998, AIP Conference Proceedings 459, edited by H. W. K. Cheung and J. N. Butler (AIP, Woodbury, NY, 1999), p. 334.

[35] LASS Collaboration, D. Aston et al., Nucl. Phys. B296, 493 (1988).

[36] A. V. Anisovich and A. V. Sarantsev, Phys. Lett. B 413, 137 (1997).

[37] OMEGA Spectrometer Collaboration, T. Armstrong et al., Nucl. Phys. B221, 1 (1983).

[38] CLEO Collaboration, B. H. Behrens et al., Phys. Rev. Lett. 80, 3710 (1998), updated by J. Alexander at 29th International Conference on High Energy Physics, Vancouver, 23-31 July 1998. 
[39] I. Halperin and A. Zhitnitsky, Phys. Rev. D 56, 7247 (1997); Phys. Rev. Lett. 80, 438 (1998); F. Yuan and K.-T. Chao, Phys. Rev. D 56, R2495 (1997); D. Atwood and A. Soni, Phys. Lett. B 405, 150 (1997); Phys. Rev. Lett. 79, 5206 (1997); H. Fritzsch, Phys. Lett. B 415, 83 (1997); H.-Y. Cheng and B. Tseng, Phys. Lett. B 415, 263 (1997); A. L. Kagan and A. A. Petrov, UCHEP-27/UMHEP-443, hepph/9707354 (unpublished); A. Ali and C. Greub, Phys. Rev. D 57, 2996 (1998); W.-S. Hou and B. Tseng, Phys. Rev. Lett. 80, 434 (1998); A. Datta, X.-G. He, and S. Pakvasa, Phys. Lett. B 419, 369 (1998).

[40] CLEO Collaboration, G. Brandenburg et al., Phys. Rev. Lett. 75, 3804 (1995).

[41] In particular, we find no difficulty in fitting the decay $D_{s} \rightarrow \pi^{+} \eta^{\prime}$ without disconnected diagrams, in contrast to the conclusions of Refs. [24] and [25]. However, the likelihood that this process does receive contributions from such diagrams indicates that the agreement we obtained with the observed rate for $D_{s} \rightarrow \bar{K}^{0} K^{+}$may be fortuitous.

[42] See, e.g., B. Blok and M. Shifman, in Third Workshop on Tau-Charm Factory, Marbella, Spain, 1-6 June 1993, edited by J. Kirkby and R. Kirkby (Editions Frontières, Gif-sur-Yvette, France, 1994), p. 247.

[43] J. L. Rosner, Phys. Lett. B 379, 267 (1996), and references therein.

[44] M. Neubert, Int. J. Mod. Phys. A 11, 4173 (1996); in Flavor Physics (Proceedings of the Fourth International Conference on Flavor Physics, KEK, Tsukuba, Japan, 29-31 October 1996), edited by Y. Kuno and M. M. Nojiri, Nucl. Phys. B Proc. Suppl. 59, 101 (1997); M. Neubert and C. Sachrajda, Nucl. Phys. B483, 339 (1997); in Heavy Flavours II, edited by A. J. Buras and M. Lindner (World Scientific, Singapore, 1998), p. 239.

[45] B. Blok, M. Gronau, and J. L. Rosner, Phys. Rev. Lett. 78, 3999 (1997).

[46] M. Gronau and J. L. Rosner, Phys. Rev. D 58, 113005 (1998).

[47] M. Ciuchini, E. Franco, G. Martinelli, and L. Silvestrini, Nucl. Phys. B501, 271 (1997); M. Ciuchini, R. Contino, E. Franco, G. Martinelli, and L. Silvestrini, Nucl. Phys. B512, 3 (1998).

[48] CLEO Collaboration, T. Bergfeld et al., Phys. Rev. Lett. 81, 272 (1998).

[49] M. Bander, D. Silverman, and A. Soni, Phys. Rev. Lett. 43, 242 (1979). 